\title{
Paradigmas en contabilidad que impiden la incorporación de aspectos diferentes del económico*
}

\author{
Accounting paradigms that impede the incorporation of non-economic aspects \\ Paradigmas contábeis que impedem a incorporação de diferentes aspectos do econômico
}

\author{
Valeria Rivera Quiguanás ${ }^{\mathrm{a}}$ \\ Universidad del Quindio, Colombia \\ vrivera@uniquindio.edu.co \\ ORCID: https://orcid.org/0000-0002-8359-6721 \\ Jhonatan Javier Henao Pulgarin \\ Universidad del Quindío, Colombia \\ ORCID: https://orcid.org/0000-0003-0851-3073
}

DOI: https://doi.org/10.11144/Javeriana.cc22.pcii

Recibido: 30/11/2019

Aceptado: $14 / 10 / 2020$

Publicado: 18/08/2021

\section{Resumen:}

Este artículo presenta los resultados de un estudio que tiene como finalidad comprender desde la teoría contable por qué la contabilidad es instrumentalizada desde una corriente eminentemente económica. Se realiza una evaluación de los enfoques paradigmáticos que enmarcan y limitan la contabilidad al ámbito económico y se define la verdadera orientación de la contabilidad en función de la manera en que contribuye al desarrollo sostenible. Este estudio se lleva a cabo en cuatro aspectos: enfoque del paradigma estudiado (económico, social, ambiental, multidimensional), sustento utilizado como defensa del paradigma, nivel de actualización del paradigma con relación a su vigencia y al desarrollo de la contabilidad en la cotidianidad, y efectos del paradigma en el saber contable. De los 21 paradigmas revisados 17 corresponden a un enfoque exclusivamente económico, 21 paradigmas proclaman realidades economicistas desde la base del sustento y han permanecido sin actualización, y 15 paradigmas producen efectos limitantes de la orientación contable. Se concluye que existe incapacidad de incorporar aspectos diferentes al económico cuando el contexto requiere una orientación de tipo tridimensional para la contabilidad.

Códigos JEL: M41, M49.

Palabras clave: Contabilidad, desarrollo sostenible, orientación contable, paradigmas contables, teoría contable.

\begin{abstract}
:
This article presents the results of a study that aims to understand, based on accounting theory, why accounting is instrumentalized from an eminently economic perspective. An evaluation is made of the paradigmatic approaches that frame and limit accounting in the economic sphere, and the true orientation of accounting is defined according to the way in which it contributes to sustainable development. This study is carried out in four aspects: focus of the studied paradigm (economic, social, environmental, multidimensional), support used as defense of the paradigm, level of updating of the paradigm in relation to its current validity and to the development of accounting in everyday life, and effects of the paradigm on accounting knowledge. Of the 21 paradigms reviewed, it is found that 17 of them have an exclusively economic focus; 21 paradigms proclaim economistic realities from the base of support and have remained without updating; and 15 paradigms produce limiting effects on the orientation of accounting. It is concluded that there is an inability to incorporate non-economic aspects when the context requires a three-dimensional orientation for accounting.
\end{abstract}

JEL Codes: M41, M49.

Keywords: Accounting, sustainable development, accounting guidance, accounting paradigms, accounting theory.

\section{Resumo:}

Este artigo apresenta os resultados de um estudo que visa compreender a partir da teoria contábil porque a contabilidade é instrumentalizada a partir de uma tendência eminentemente econômica. Procede-se a uma avaliação das abordagens paradigmáticas que enquadram e limitam a contabilidade na esfera económica e define-se a verdadeira orientação da contabilidade de acordo com a forma como contribui para o desenvolvimento sustentável. Este estudo é realizado em quatro aspectos: enfoque do paradigma estudado (econômico, social, ambiental, multidimensional), suporte utilizado como defesa do paradigma, nível de atualização do paradigma em relação à sua validade e o desenvolvimento da contabilidade na vida cotidiana, e efeitos do paradigma

Notas de autor

a Autora de correspondencia. Correo electrónico: vrivera@uniquindio.edu.co 
no conhecimento contábil. Dos 21 paradigmas revisados, obtém-se que 17 deles têm enfoque exclusivamente econômico; 21 paradigmas proclamam realidades econômicas a partir do sustento e permaneceram sem atualização; e 15 paradigmas produzem efeitos limitantes da orientação contábil. Conclui-se que há incapacidade de incorporação de aspectos outros que não os econômicos quando o contexto exige uma orientação tridimensional da contabilidade.

Códigos JEL: M41, M49.

Palavras-chave: Contabilidade, desenvolvimento sustentável, orientação contábil, paradigmas contábeis, teoria contábil.

\section{Introducción}

La contabilidad está sujeta a infinidad de paradigmas como cualquier otro conocimiento. Dichos enfoques paradigmáticos aceptados han propiciado una variedad de concepciones sobre el objeto de estudio y propósito del saber. Esta discrepancia genera necesidad de investigar sobre los paradigmas en contabilidad que impiden la incorporación de aspectos diferentes del económico, ya que actualmente la orientación contable se halla limitada en este único enfoque, aun cuando el contexto demanda el reconocimiento contable de una realidad que en verdad contribuya a la "sostenibilidad integral de la riqueza en todas sus dimensiones" (Mejía, Montilla, Montes, \& Mora, 2015, p. 22).

El reduccionismo, la limitación o poca capacidad -o incapacidad- de respuesta a crisis ambientales y sociales deben ser combatidos desde la investigación contable como factor de desarrollo académico y científico, que propenda por una cultura de debate enmarcada desde el sentido de pertenencia por el saber, generando así estándares contables ambientales y sociales óptimos a ser aplicados en el mundo globalizado. Por lo tanto, se hace necesario identificar qué está obstaculizando la contribución de la contabilidad al desarrollo ambiental, social y económico, indicando aquellos enfoques paradigmáticos que la enmarcan desde la esfera económica.

En esta investigación se destacan los siguientes autores en tres pilares representados en los conceptos de Paradigma desde la óptica de Kuhn (1962) y Viveros (2015), Desarrollo Sostenible desde la ONU (1983) y Mejía, Montilla, Montes y Mora (2014) y Contabilidad bajo el concepto de García (1997). Además, de otros autores como Bunge (1985) con las características de la ciencia, Hendriksen (1974), Belkaoui (1993), Tua (1996), Mantilla y Tristancho (1997) con paradigmas contables. Su encuentro en un marco teórico permite abordar los paradigmas contables desde una perspectiva de análisis crítico frente a los enfoques que cada uno representa de manera individual, es decir, económico, social, ambiental o multidimensional.

En este artículo se pretende explicar los enfoques paradigmáticos que enmarcan la contabilidad únicamente desde la esfera económica y/o complementaria de otras dimensiones. Para ello, inicialmente se examinan los paradigmas que limitan o no su orientación solo a la dimensión económica a través de un análisis en términos de enfoque, base del sustento, nivel de actualización y efectos generados, para finalmente definir su verdadera orientación desde el punto de vista teórico en función a su contribución al desarrollo sostenible.

Lo anterior debido a que la contabilidad observada como una herramienta o instrumento al servicio de la economía se limita en su capacidad de responder a crisis ambientales y sociales, impidiendo que se conciba como "un saber al servicio de la protección del interés nacional" (Mejía \& Montes, 2009, pág. 149). Así, se llega a la base originaria del conocimiento contable que entorpece la incorporación de dimensiones diferentes de la económica, entendida como aquellos paradigmas del saber contable que sustentan este conocimiento como instrumento de la economía y modelo económico imperante (capitalismo) en su proceso de acumulación de riqueza y especulación. Igualmente, se evidencia cómo la condición de impedir o limitar a través del paradigma conlleva a desvirtuar la verdadera orientación de la contabilidad.

En consecuencia, se sienta una base de línea teórica que permite desarrollar posteriores investigaciones orientadas a la ampliación y aceptación general del objeto de estudio de la Contabilidad, ya sea a través de la evaluación y reestructuración de los paradigmas dominantes o a través del surgimiento de otros orientados al interés general y el desarrollo íntegro (no solo económico) de las naciones. 


\section{Marco teórico}

Dentro del referente teórico sobre paradigmas contables se encuentran los aportes de autores de mayor relevancia que una vez evaluados, permiten profundizar en las relaciones existentes sobre los pilares de paradigmas, desarrollo sostenible y contabilidad. A continuación, se relacionan los aportes teóricos consultados que para efectos de mayor comprensión han sido clasificados en postulados teóricos sobre paradigmas, desarrollo sostenible, contabilidad, contabilidad en las dimensiones ambiental, social y económica, y paradigmas contables.

\section{Paradigmas}

Kuhn (1962) define el concepto de ciencia normal como una investigación basada en realizaciones científicas que ya han sido aceptadas por la comunidad científica; dichas realizaciones científicas constituyen lo que denomina paradigmas. Más exactamente describe el término como "realizaciones científicas universalmente reconocidas que, durante cierto tiempo, proporcionan modelos de problemas y soluciones a una comunidad científica" (1962, p. 13). Según este autor el paradigma es reconocido durante "cierto tiempo" hasta que este no tenga la capacidad de responder a todo lo que se conoce. Es decir, hasta que se presenta una revolución científica donde los nuevos resultados de investigación demuestran que los modelos de explicación de la realidad antes concebidos son inaceptables y por consiguiente el conocimiento debe evolucionar con el establecimiento de nuevos paradigmas.

Daros (2007) sostiene que el pensamiento de Kuhn y Popper no era del todo contradictorio, pues llegaron a algunas conclusiones similares. Para ellos la ciencia no progresa por acumulación, sino por una revolución científica donde una vieja teoría puede ser reemplazada por una teoría opuesta que surge en respuesta a los retos del contexto.

Con respecto al lineamiento de Kuhn, Montes, Mejía, \& Valencia (2006) establecen que la contabilidad no alcanza los supuestos de la ciencia normal y que no hay comunidad científica que no actúe en referencia a un paradigma, por tanto se hace fundamental lo dicho por Lakatos citado por García (2008):

En un programa de investigación progresivo, la teoría conduce a descubrir hechos nuevos hasta entonces desconocidos. En los programas regresivos, las teorías son fabricadas sólo para acomodar los hechos ya conocidos reafirmando el programa de investigación progresivo en el que se debe hayar la contibilidad actualmente para obtener una mayor fundamentación de su nueva realidad. (García, 2008, p. 196).

De acuerdo con Viveros (2015), el paradigma ha evolucionado de una concepción singular a una estructura plural o matriz disciplinar de Kuhn. La singularidad paradigmática consiste en la construcción de soluciones de manera particular o única. La pluralidad matricial consiste en dar soluciones a traves de paradigmas articulados, es decir, se abre la posibilidad de comunicarse entre diferentes paradigmas para solucionar realidades concretas. En contexto, la actualidad de diversos saberes empieza a reconocer la necesidad de adoptar la matriz disciplinar como noción útil para dar respuesta al desarrollo sostenible, sin embargo, se requiere de paradigmas nuevos para el aspecto social y ambiental que los articulen de manera efectiva.

\section{Desarrollo sostenible}

El contexto del siglo XXI ha generado una demanda específica a la contabilidad en relación con su aporte en aspectos ambientales y sociales que requiere de conceptualización, medición, valoración, registro, revelación y control de este tipo de riqueza; hecho que recae en la evaluación de sus paradigmas. Ejemplo de esta demanda es el pronunciamiento de la ONU (1983) que promueve el desarrollo sostenible con el deber y compromiso 
de cada individuo para cuidar y preservar el planeta para las generaciones futuras buscando un desarrollo que reduzca, elimine y/o controle los posibles daños ocasionados al mismo.

Para lo anterior es importante reconocer la discusión que se genera respecto a la utilización del concepto desarrollo sostenible o sustentable, que "si bien tienen aristas comunes, lo sustentable hace relación a la armonía existente entre lo económico, lo social, lo ambiental con el sistema de valores, en tanto que lo sostenible considera cada uno de dichos subsistemas por separado" (Zarta, 2018, p. 409). Sin embargo, esta discusión puede ser objeto de otro estudio.

\section{Contabilidad}

En respuesta al concepto de desarrollo sostenible la contabilidad demanda un cambio de orientación, para su comprensión se hace necesario partir del concepto que se aplica actualmente. Desde su concepción la contabilidad se ubicó dentro de las ciencias económicas, donde su objeto material es la realidad (socio) económica, su objeto formal es el conocimiento cualitativo y cuantitativo de la misma y su fin es determinar la situación de la unidad económica a través de la captación, medida, valoración, representación y comunicación de la información generalmente cuantitativa para la toma de decisiones (Uribe, 2014, p. 245).

García (1997, p. 13) sustenta un amplio postulado teórico de contabilidad representado en la superación de la visión economicista, para lo cual sostiene que la contabilidad es una ciencia de carácter factual, cultural y aplicada que estudia las interrelaciones que se presentan entre los componentes de los diferentes hechos informativos de las diversas clases de organizaciones. En concordancia, se defiende la postura de ciencia para la contabilidad realizando un comparativo con las características que según Bunge (1985) debe tener la ciencia. Su perspectiva adscribe a la 'ciencia' contable dentro del grupo de las ciencias factuales; es decir, al mismo nivel de la economía, la sociología, la biología, entre otras, y se hace evidente la necesidad de ampliar la orientación de la contabilidad.

Por otra parte, García (2002) estructura unas bases para formular la teoría general contable con sus respectivos elementos y manifiesta que en el enfoque científico de la contabilidad es nuevo el desarrollo de modelos alternativos para los elementos que constituyen la disciplina que se centran en lo patrimonial o financiero, principalmente en los informes financieros. De ahí que la Contabilidad está siendo observada desde una concepción reduccionista. La orientación del saber se ha limitado a la simple incorporación de aspectos económicos, excluyendo otras realidades que participan también dentro del contexto en el que se desarrolla el conocimiento contable y que previamente ya se han identificado como necesarias, dando paso a la teoría tridimensional de la contabilidad.

\section{Contabilidad en las dimensiones ambiental, social y económica}

Mejía (2014) establece que la contabilidad es una ciencia social aplicada y define una amplia orientación del saber contable en lo que denomina Teoría Tridimensional de la Contabilidad (T3C). Esta propuesta sistematiza e integra la valoración integral (cualitativa y cuantitativa) de la riqueza ambiental, social y económica, desde el punto de vista de una estructura común y unificada, pero que a la vez diferencia las especificidades de las tres realidades que incluye.

De otra parte, Mejía (2016), defiende la Contabilidad para la Sostenibilidad, ofreciendo una fuerte crítica a dos conceptos: a) desarrollo, que está relacionado o adscrito al crecimiento económico, y b) sustentabilidad, que no tiene cabida por considerarse temporal. Su proposición se basa en una contabilidad tridimensional que da respuesta a las tres realidades para la sostenibilidad (tabla 1). 
TABLA 1

Sistemas de la contabilidad para la rendición de cuentas en función de la sostenibilidad

\begin{tabular}{|l|l|}
\hline \multicolumn{1}{|c|}{ Realidad } & \multicolumn{1}{c|}{ Sistema Contable } \\
\hline Ambiental & Biocontabilidad \\
\hline Social & Sociocontabilidad \\
\hline Económica & Contabilidad económica \\
\hline
\end{tabular}

Fuente: elaboración propia según Mejía (2016).

De acuerdo con Amigo (2018) el sistema contable para la realidad social y ambiental bajo la perspectiva reduccionista mantiene el enfoque económico para un hecho que presenta otra naturaleza. La contabilidad social y ambiental busca generar información que incorpore más aspectos para satisfacer necesidades de información para la toma de decisiones, evidenciando resultados ambientales y sociales, pero principalmente económicos, asociados a procesos productivos.

Por otro lado, de acuerdo con Mejía, Montilla, Montes, \& Mora (2015) el sistema contable, bajo un enfoque tridimensional para la realidad social, define la sociocontabilidad y la biocontabildad como una disciplina social y ambiental que busca estudiar la valoración cualitativa y cuantitativa de la existencia y circulación de la riqueza social y ambiental. Para ello, hacen uso de diversos métodos que permitan evaluar y controlar la gestión de las organizaciones sobre dichas riquezas. Ambos conceptos se fundamentan bajo un nuevo enfoque.

Mejía, Montes y Mora (2013) pretende establecer un nuevo paradigma entre la comunidad científica contable que propende por ampliar el concepto de contabilidad permitiendo la presentación de estados contables integrales que rindan cuenta de lo social, ambiental y económico, e identificar el objeto de estudio de la contabilidad donde el aspecto material se refiere a la riqueza controlada por las organizaciones y lo formal a la valoración cualitativa y cuantitativa de esta riqueza en las tres realidades. De esta manera se supera la visión reduccionista del saber al definirla como ciencia social aplicada capaz de ser autónoma e independiente principalmente de la ciencia económica y se reconoce la posibilidad de valoraciones distintas a la unidad de medida monetaria.

De estos modelos se desprenden los sistemas contables de Biocontabilidad, Sociocontabilidad y Contabilidad Económica. Partiendo de esto, se identifica que el fin genérico de la Contabilidad según la T3C es "contribuir a la acumulación, generación, distribución y sostenibilidad integral de la riqueza ambiental, social y económica controlada por las organizaciones" (Mejía, Montilla, Montes, \& Mora, 2014, p. 102).

Finalmente, de acuerdo con Ariza (2000), la contabilidad como saber que permite la rendición de cuentas de las relaciones técnicas de producción y las relaciones sociales de distribución de la riqueza, puede contribuir al desarrollo sostenible solo si se está en la capacidad de generar estructuras conceptuales que permitan a las organizaciones en la práctica, dar cuenta sobre los diferentes tipos de riqueza que controlan (ambiental, social, económica), situación que requiere importantes transformaciones.

\section{Paradigmas contables}

A efectos de evaluar la capacidad que tiene la contabilidad de incorporar aspectos diferentes del económico resulta necesario contrastar los paradigmas aceptados con base en el libro Paradigmas en Contabilidad de Montes, Mejía, \& Valencia (2006). Su investigación permite identificar 21 diferentes paradigmas sobre contabilidad conocidos de 1974 a 2006 y que se han seguido desarrollando. Además, permite analizar el alcance y el enfoque de los mismos.

Según Montes, Mejía, \& Valencia (2006), Hendriksen proporciona 11 enfoques, a saber: a) Razonamiento deductivo, establece declaración de postulados de contabilidad concernientes al medio económico y la aplicación de principios a situaciones específicas. b) Enfoque inductivo, argumenta que sí es posible hallar relaciones recurrentes con las que pueden formularse generalizaciones y principios. c) Enfoque ético, 
manifiesta un interés por la inclusión del campo ético que corresponde a un principio de convivencia para la sociedad y sostiene que los informes financieros deben presentar una declaración verdadera y exacta de los hechos. d) Enfoque en teoría de la comunicación, representa la contabilidad como un sistema de información o comunicación de datos útiles. e) Enfoque conductual, hace énfasis en determinar los comportamientos económicos de los transactores del mercado. f) Enfoque sociológico, incorpora aspectos sociales dentro de lo que llama comportamiento empresarial. g) Enfoque macroeconómico, relacionado con políticas económicas nacionales. h) Enfoque no pragmático, refleja la orientación netamente teórica de la contabilidad. i) Enfoques no teóricos, hacen alusión al aspecto práctico de la contabilidad como sistema de información empresarial. j) Enfoque en teoría de las cuentas, reflexiona sobre la necesidad de utilizar códigos que carguen saldos y cumplan una ecuación patrimonial universal. k) Enfoques eclécticos de teoría de la contabilidad, se refieren a la posibilidad de combinar de diversas maneras los enfoques anteriores cuando es necesario para una adecuada información contable.

Posteriormente, Belkaoui (1993) presenta seis paradigmas contables: a) Antropológico - Inductivo, referido al planteamiento de principios contables producto de la experiencia específica de entidades y sus comportamientos gerenciales; b) Deductivo - Beneficio verdadero, busca la mejor forma de determinación de resultados producto de aplicación de principios generalizados y con los mejores métodos de reconocimiento y medición; c) Utilidad decisión - Modelo de decisión, trata de la utilidad de la información contable para tomar decisiones acertadas; d) Utilidad decisión - Comportamiento agregado del mercado, considera la utilidad de la información contable para descifrar conductas que presente el mercado especulativo del capital; e) Utilidad decisión - Usuario individual, refleja la utilidad de la información contable para el humano individual permeado por conductas del mercado y sus propios intereses; y f) Economía - Información, evalúa la relación costo beneficio de obtener información contable que permita la toma de decisiones adecuadas.

Es importante señalar que sobre la perspectiva de Belkaoui (1993) existen señalamientos como el de Martínez (2007) que indican que el diseño multiparadigmático es la representación del empirismo, estructuras hipotéticas y herramientas básicas de descripción de hechos organizacionales.

Este trabajo sostiene un abordaje representado en la evaluación individual de los enfoques paradigmáticos objeto de estudio. En ese sentido, se someten singularmente a consideración los enfoques ya mencionados de Belkaoui, por supuesto, dejando constancia y respetando la originalidad planteada por el autor de efectuar un abordaje a partir del enfoque multiparadigmático.

Tua (1996) describe la contabilidad entre dos grandes paradigmas existentes: (i) el paradigma del beneficio verdadero o la ganancia líquida, que busca la verdad económica de los resultados obtenidos al final de un proceso contable (Montes, Mejía, \& Valencia, 2006). Su objetivo es la generación del dato del beneficio real obtenido en un periodo visualizado desde un marco jurídico (Santos, Durán G, Urrea Bello, \& Urquijo, 1997). Y (ii) el paradigma de utilidad para la toma de decisiones, que se refiere la necesidad de los usuarios de la información contable en sentido propio de acertar en la consecución de sus intereses.

De otro lado, Mantilla y Tristancho (1997) relacionan tres paradigmas que otorgan un sustento al enfoque de utilidad para la toma decisiones: paradigma de protección patrimonial, paradigma para la toma de decisiones y paradigma formalizado. A modo de mayor actualidad, Tua (2012) manifiesta para este paradigma que "bajo el mismo, la Contabilidad no trata tanto de medir en abstracto unos hechos pasados, en búsqueda de un concepto único y autosuficiente de verdad económica, sino de medir e informar para la toma de decisiones" (Tua, 2012, p. 100).

Posteriormente, Montes, Mejía y Valencia (2006) identifican dos paradigmas: (i) Bursátil - Interés público, que considera el modelo económico imperante en la sociedad actual que rige en sentido de especulación financiera, y (ii) Ético socio-humanístico, que supone una propuesta de ruptura paradigmática que requiere el reconocimiento del medio ambiente, la sociedad y la economía como las tres dimensiones sobre las que deben rendir cuenta las organizaciones del mundo actual. 
Ahora, desarrollados los paradigmas objeto de estudio, cabe resaltar que estos han generado cuestionamientos múltiples por parte de la investigación contable, entre los que se puede destacar, de acuerdo con Casal \& Viloria (2007) que la ciencia contable responde de manera fiel a los paradigmas positivistas como el funcionalismo y el estructuralismo, proporcionando los rasgos y características actuales de los sistemas contables, el tratamiento de cuentas en los estados financieros, el paso a paso del ciclo contable, los métodos para auditoria y lo concerniente al costo y la utilidad, etc.

\section{Método}

El trabajo tiene un enfoque cualitativo propio de la investigación en ciencias sociales. El estudio es de tipo descriptivo-documental, ya que a través de la compilación de manuscritos teóricos enuncia una serie de rasgos y características entre los ejes principales: paradigmas, desarrollo sostenible y contabilidad. El método para la obtención del conocimiento es el análisis, pues inicia por la identificación de cada una de las partes que caracterizan una realidad y establece relación causa efecto a través de la revisión teórica bajo unos criterios establecidos; este método se utiliza en la evaluación de los 21 paradigmas de manera individual. Posteriormente, el método inductivo permite que de verdades particulares se concluyan verdades generales por las propiedades comunes de los casos estudiados, método utilizado para las conclusiones e identificación de la verdadera orientación de la contabilidad.

El análisis de la información se realizó en dos momentos. El primer momento corresponde a la evaluación de los enfoques paradigmáticos que enmarcan y limitan la contabilidad en el ámbito económico y análisis individual de los 21 paradigmas contables objeto de estudio: 11 paradigmas de Hendriksen, seis de Belkaoui, dos de Tua y dos de Montes, Mejía y Valencia, con relación a cuatro aspectos relevantes para cada uno: a) el enfoque que contiene el aspecto económico, social, ambiental y multidimensional porque determina la orientación del paradigma; b) la base del sustento (teorías) utilizado como defensa porque contrasta las realidades que promulga y validar el enfoque; c) el nivel de actualización con relación a la capacidad de respuestas del paradigma a la cotidianidad de la contabilidad porque evalúa la vigencia, y d) los efectos o implicaciones que genera reconocer o aceptar el paradigma evaluado porque definen la capacidad de incorporar aspectos ambientales y sociales. Para el análisis, cada elemento cuenta con una descripción y forma de interpretación, con el fin de que el proceso de revisión teórica sea efectuado desde una concepción uniforme que permita comparar los paradigmas estudiados sobre la base de una misma perspectiva lógica.

En primer lugar, el enfoque del paradigma estudiado describe si dicho paradigma sustenta la contabilidad en un enfoque económico, social, ambiental, o si adopta la relación de diferentes enfoques (multidimensional) bajo el concepto de desarrollo sostenible. De acuerdo con Bustillo y Martínez (2008, p. 391) el enfoque se refiere al modelo con el cual se interpreta la realidad que puede encontrar concreción en diferentes espacio de acuerdo con la visión de quien dilucide. En ese sentido, el criterio de análisis del resultado obtenido luego de la aplicación del instrumento de observación puede ofrecer cuatro diferentes formas de interpretación:

- Enfoque económico: solo incorpora aspectos económicos.

- Enfoque social: solo incorpora aspectos sociales.

- Enfoque ambiental: solo incorpora aspectos ambientales.

- Enfoque multidimensional: incorpora dos o más aspectos (económico, ambiental, social).

En segundo lugar, la base del sustento utilizado como defensa del paradigma estudiado describe los argumentos expuestos desde la percepción de saber contable que son utilizados en el paradigma para dar sustento al enfoque que proclama este mismo, realizándose el análisis de manera individual teniendo en cuenta que según Marín A. (2007, p. 39) cada paradigma es excluyente y único. En ese sentido, el criterio de análisis puede ofrecer dos diferentes formas de interpretación de los argumentos expuestos por el paradigma para dar 
sustento a su enfoque: a) proclaman realidades contundentes en relación con el contexto que abarca el saber contable, y b) no proclaman realidades contundentes en relación con dicho contexto.

Entiéndase realidades contundentes como aquellas que son manifestadas en la argumentación del paradigma evaluado, que fácilmente pueden ser observadas y verificadas con pruebas, impidiendo la generación de duda alguna respecto de lo que se afirma.

En tercer lugar, el nivel de actualización del paradigma estudiado describe si se encuentra vigente la capacidad que tiene el paradigma de responder a los contextos actuales sobre los que se desempeña el saber contable, pues en los períodos de ciencia normal esta crece y se desarrolla en forma acumulativa, de manera que cuando un enigma no puede resolverse, la teoría entra en crisis, y en ocasiones se genera un cambio de paradigma Marín G. (2007), requiriendo de una actualización. En ese sentido, el criterio de análisis puede ofrecer dos diferentes formas de interpretación: a) el paradigma estudiado sigue vigente para el desarrollo de la contabilidad, o b) el paradigma estudiado no sigue vigente para tal fin.

En cuarto y último lugar, los efectos que genera el paradigma estudiado evalúan la aceptación del paradigma y las repercusiones que esto ocasiona en el desenvolvimiento de la realidad tridimensional (ambiente, sociedad y economía) teniendo en cuenta que de acuerdo con Marín A. (2007) el paradigma crea la impresión de lo que es verdadero. El criterio de análisis del resultado puede ofrecer dos diferentes formas de interpretación: a) el paradigma estudiado genera incorporación de aspectos diferentes del económico en el desarrollo del saber contable, o b) el paradigma analizado no genera dicha incorporación.

Y el segundo momento, corresponde a la definición de la verdadera orientación de la contabilidad en función de la manera en que contribuye al desarrollo sostenible; en esta parte se aplica el método inductivo al evaluar los hallazgos y concluir de manera generalizada.

\section{Resultados}

Los resultados obtenidos se dividen en dos secciones. Los paradigmas contables que impiden o no incorporan aspectos diferentes al económico desde el análisis de los 21 paradigmas objeto de investigación, y la verdadera orientación de la contabilidad en función de su aporte al desarrollo sostenible.

\section{Paradigmas contables que impiden o no incorporar aspectos diferentes al económico}

Para esta sección se inicia con la identificación de superposiciones, que relaciona los paradigmas objeto de estudio bajo dos enfoques agrupadores y se finaliza con el análisis individual de estos mismos paradigmas para concluir sobre su capacidad de incorporar o no aspectos diferentes al económico. En primer lugar, se establece relación entre las perspectivas de los diferentes autores a efectos de evitar superposiciones en los enfoques evaluados (ver la tabla 2). 
TABLA 2

Identificación de superposiciones: relación entre paradigmas estudiados

\begin{tabular}{|c|c|c|c|}
\hline \multirow[b]{2}{*}{ Autores } & \multicolumn{2}{|c|}{ Tua Pereda } & \multirow[b]{2}{*}{$\begin{array}{l}\text { Sin paradigma } \\
\text { agrupador }\end{array}$} \\
\hline & \begin{tabular}{|l|} 
Paradigma agrupador \\
Beneficio verdadero o \\
Ganancia Liquida \\
\end{tabular} & $\begin{array}{l}\text { Paradigma agrupador } \\
\text { Utilidad para la toma de } \\
\text { decisiones }\end{array}$ & \\
\hline \multirow{9}{*}{ Hendriksen } & Razonamiento deductivo & $\begin{array}{l}\text { Enfoque basado en la teoría } \\
\text { de la comunicación }\end{array}$ & \multirow{13}{*}{ No aplica } \\
\hline & Enfoque inductivo & $\begin{array}{l}\text { Enfoque basado en la } \\
\text { conducta }\end{array}$ & \\
\hline & No aplica & Enfoque macroeconómico & \\
\hline & \multicolumn{2}{|l|}{ Enfoque ético } & \\
\hline & \multicolumn{2}{|l|}{ Enfoque sociológico } & \\
\hline & \multicolumn{2}{|c|}{ Enfoque en teoría de las cuentas } & \\
\hline & \multicolumn{2}{|c|}{ Enfoques eclécticos de teoría de la contabilidad } & \\
\hline & \multicolumn{2}{|l|}{ Enfoque no pragmático } & \\
\hline & \multicolumn{2}{|l|}{ Enfoque no teórico } & \\
\hline \multirow{4}{*}{ Belkaoui } & Antropológico - inductivo & $\begin{array}{l}\text { Utilidad decisión - Modelo } \\
\text { de decisión } \\
\text { agregado del mercado }\end{array}$ & \\
\hline & \begin{tabular}{|l|} 
Deductivo - beneficio \\
verdadero
\end{tabular} & $\begin{array}{l}\text { Utilidad decisión - } \\
\text { comportamiento }\end{array}$ & \\
\hline & No aplica & $\begin{array}{l}\text { Utilidad decisión - Usuario } \\
\text { individual }\end{array}$ & \\
\hline & No aplica & Economía - información & \\
\hline $\begin{array}{l}\text { Montes, } \\
\text { Mejía y } \\
\text { Valencia }\end{array}$ & No aplica & Bursátil - Interés público & $\begin{array}{l}\text { Paradigma ético } \\
\text { socio-humanístico }\end{array}$ \\
\hline
\end{tabular}

Fuente: elaboración propia. El paradigma ético socio-humanístico se propone con la noción de beneficio verdadero más no desde el contexto económico sino de carácter tridimensional.

Teniendo en cuenta lo que representa la aceptación de las posiciones de Hendriksen (1974), Belkaoui (1993) y Montes, Mejía y Valencia (2006), se establece la relación entre los paradigmas estudiados identificando dos paradigmas agrupadores profundizados por Tua (1996): paradigma agrupador del Beneficio verdadero o Ganancia líquida y paradigma agrupador de Utilidad para la toma de decisiones.

Para Hendriksen, el paradigma agrupador Beneficio verdadero o Ganancia líquida se relacionan con el Razonamiento deductivo y el Enfoque inductivo porque más allá de que el primero concluya a partir de lo general y sea producto de un análisis dado sobre condiciones de economía mundial y nacional, y que el segundo generalice a partir de lo especifico y sea producto de un análisis de unidades económicas representadas por empresas; siempre conservan el objetivo de llegar a principios económicos para hacer contabilidad y lograr la presentación de unos estados financieros de representación fiel en sus resultados. Para el paradigma agrupador Utilidad para la toma de decisiones se relacionan los enfoques basados en la teoría de la comunicación y en la conducta, y el macroeconómico, debido a que se sustentan en la producción de información financiera oportuna que permita transmitir un mensaje de la situación que se vive y de los comportamientos organizacionales que han causado dicha situación. Por último, los enfoques ético, sociológico, teoría de las cuentas, teoría de la contabilidad, no pragmático y no teórico, guardan relación con los dos paradigmas agrupadores porque para estos el fin económico de la contabilidad es rendir cuenta de los resultados y que logren influenciar la decisión de los usuarios de la información.

En cuanto a Belkaoui, para el paradigma agrupador Beneficio verdadero relaciona los enfoques Antropológico - inductivo y Deductivo - beneficio verdadero porque el primero busca establecer teoría contable a partir del conocimiento económico de la empresa, para que así, el segundo, defienda el beneficio económico como eje central de la información contable. Para el paradigma agrupador Utilidad para la toma de decisiones se relacionan Utilidad decisión - modelo de decisión agregado del mercado, Utilidad decisión - comportamiento, Utilidad decisión - usuario individual y Economía - información porque profundizan en lo útil que debe ser la preparación y presentación de información contable para que los usuarios del mercado puedan tomar decisiones de carácter económico basado en el comportamiento financiero que resume la contabilidad de una organización. 
Para Montes, Mejía y Valencia no aplica el paradigma agrupador Beneficio verdadero o Ganancia Líquida debido a que sus postulados se basan en la proposición de dos nuevos enfoques paradigmáticos a modo de revolución científica de un saber contable que amplíe su objeto de estudio, reconociendo la sociedad y el medio ambiente como realidades sobre las que también se debe responder organizacionalmente y rendir cuentas, pasando de la Ganancia líquida a la Ética biocéntrica de la contabilidad. Para el paradigma agrupador Utilidad para la toma de decisiones, se relaciona el enfoque Bursátil - interés público porque enfatiza en la necesidad de información que permita confianza en sentido de orientación económica especulativa que permita satisfacción de los intereses del usuario bursátil. Finalmente, sin paradigma agrupador, se encuentra la propuesta de paradigma ético socio-humanístico porque a diferencia de los ya señalados, este busca defender para la contabilidad las orientaciones del desarrollo sostenible en sus tres categorías producto de la relación naturaleza-hombre-economía, permitiendo una sensibilización a la hora de rendir cuentas en lo ambiental y social.

En síntesis, para el análisis por autores se tiene que Hendriksen trabaja bajo un estudio del paradigma individual, es decir analizó 11 enfoques de la contabilidad de manera específica. Belkaoui, por su parte, trabaja bajo el estudio multiparadigmático, ya que observa el proceso contable a través de la existencia de seis enfoques que persiguen la máxima aceptación en la comunidad contable y que se resumen en los objetivos de la búsqueda del verdadero resultado, el facilitamiento de información útil para tomar decisiones y la economía de la información. Y Montes, Mejía y Valencia utilizan el análisis integrador que quiere decir el reconocimiento de un contexto de actuación del saber contable delimitado por la relación medio ambienteeconomía-sociedad.

A la hora de profundizar en materia de incorporación de aspectos diferentes del económico en el saber contable se debe considerar que existen múltiples visiones paradigmáticas sustentadas para la contabilidad, por lo cual es necesario identificar para cada paradigma encontrado elementos comunes considerados como pertinentes y relevantes en su análisis. Para ello se revisaron teóricamente de manera individual los paradigmas de Hendriksen, Belkaoui, Tua y Montes, Mejía y Valencia a partir de los criterios planteados (ver métodos), y se procede a determinar los paradigmas que están limitando la orientación contable (ver tabla 3).

TABLA 3

Resultados obtenidos de la revisión teórica de cada paradigma

\begin{tabular}{|c|c|c|}
\hline Paradigma & Enfoque & $\begin{array}{c}\text { Efectos: :Genera } \\
\text { incorporación (de aspectos } \\
\text { diferentes del económico)? }\end{array}$ \\
\hline Razonamiento deductivo & $\begin{array}{l}\text { Multidimensional (económico, } \\
\text { social) }\end{array}$ & Sí \\
\hline Enfoque inductivo & Económico & No \\
\hline Enfoque ético & Económico & Sí \\
\hline $\begin{array}{l}\text { Enfoque basado en teoría de la } \\
\text { comunicación }\end{array}$ & Económico & No \\
\hline Enfoque basado en conducta & Económico & No \\
\hline Enfoque sociológico & $\begin{array}{l}\text { Multidimensional (económico, } \\
\text { social) }\end{array}$ & Sí \\
\hline Enfoque macroeconómico & Económico & No \\
\hline Enfoque no pragmático & Económico & No \\
\hline Enfoques no teóricos & Económico & No \\
\hline Enfoque en teoría de las cuentas & Económico & No \\
\hline $\begin{array}{l}\text { Enfoques eclécticos de teoría de } \\
\text { contabilidad }\end{array}$ & $\begin{array}{l}\text { Multidimensional (económico, } \\
\text { social) }\end{array}$ & Sí \\
\hline Antropológico - inductivo & Económico & No \\
\hline Deductivo - Beneficio verdadero & Económico & No \\
\hline Utilidad decisión - Modelo de decisión & Económico & No \\
\hline $\begin{array}{l}\text { Utilidad decisión-Comportamiento } \\
\text { agregado del mercado }\end{array}$ & Económico & No \\
\hline Utilidad decisión - Usuario individual & Económico & No \\
\hline Economía - Información & Económico & No \\
\hline Beneficio verdadero o ganancia líquida & Económico & No \\
\hline Utilidad para toma de decisiones & Económico & No \\
\hline Bursátil - Interés público & Económico & Sí \\
\hline Ético socio-humanístico & $\begin{array}{l}\text { Multidimensional (económico, } \\
\text { social, ambiental) }\end{array}$ & Sí \\
\hline
\end{tabular}

actualización es vigente (en el desarrollo de la contabilidad).

Fuente: elaboración propia basada en Montes, Mejía y Valencia (2006). 
Los resultados obtenidos del análisis de los 21 paradigmas permiten interpretar que el enfoque únicamente económico se representa en 17 paradigmas, mientras que los cuatro restantes tienen un enfoque multidimensional. Para estos últimos, el Razonamiento deductivo, Enfoque sociológico, Enfoques eclécticos de teoría de la contabilidad relacionan el enfoque económico y social; y el Ético socio-humanístico refiere los tres enfoques (ambiental, social y económico).

Más allá de su enfoque, los 21 paradigmas examinados sí proclaman realidades contundentes dentro del contexto sobre el que se desenvuelve el saber contable. Es decir, los paradigmas analizados permiten que la contabilidad responda sobre una realidad principalmente económica en razón a que se fundamenta en la teoría contable clásica. Situación que implica que los paradigmas analizados desde la base del sustento proclaman realidades de visión economicista, limitando la incorporación de aspectos diferentes.

La totalidad de los paradigmas examinados cumplen con el nivel de actualización, es decir, los paradigmas permanecen vigentes desde la promulgación de los mismos en el desarrollo de la contabilidad económica lo que limita la incorporación de aspectos diferentes. Dicha situación, representa que a pesar del paso del tiempo y de la generación de nuevas necesidades para la contabilidad, los paradigmas han permanecido sin actualización concreta más allá de las críticas que exponen diversos autores de teoría contable emergente o contemporáneas.

De los paradigmas estudiados 15 producen efectos limitantes de la orientación contable, hecho que se traduce en no tener la capacidad de generar incorporación de aspectos diferentes del económico. Sin embargo, seis de los restantes cuentan con capacidad de incorporación, así: relación aspecto social y económico a) razonamiento deductivo, b) enfoque sociológico, c) enfoques eclécticos de teoría de la contabilidad. y relación aspecto ambiental, social y económico; a) enfoque ético socio-humanístico, b) enfoque ético, y c) enfoque bursátil - interés público.

De esta manera se determina que la mayoría de los paradigmas contables estudiados impiden o limitan la incorporación de aspectos diferentes del económico desde la interpretación del enfoque, base del sustento, nivel de actualización y efecto. Se enriquece entonces la discusión académica en términos de paradigmas contables y su influencia en la orientación de la contabilidad.

\section{Verdadera orientación de la Contabilidad}

Se identifican dos tendencias de orientaciones en la contabilidad a partir de la discusión entre la teoría contable clásica y la teoría tridimensional emergente. A partir de ellas, se evidencia que su orientación requiere un cambio de paradigma tradicional a tridimensional. La concepción contable actual reconoce una orientación meramente económica ampliamente aceptada por la comunidad científica contable y responde a necesidades de información financiera, mientras que la contabilidad desde la propuesta de T3C de Mejía, Montilla, Montes y Mora (2015) reconoce una orientación para la interacción de las tres dimensiones del desarrollo sostenible: ambiente, sociedad y economía que atiende a la necesidad de información contable íntegra útil para la sostenibilidad integral de la riqueza y la toma de decisiones en las tres realidades.

Ahora bien, la diferencia entre las dos orientaciones radica en el reconocimiento del contexto sobre el que se desarrolla la contabilidad. Más allá de la posición dominante de la orientación económica (teoría clásica) sobre la orientación tridimensional (teoría tridimensional) en la contabilidad, la definición de la verdadera orientación del saber contable debe responder a un contexto de realidad contundente (observable y verificable). Situación que hace necesario recordar dos aspectos: que la contabilidad puede contribuir al desarrollo sostenible; y que si la contabilidad puede contribuir al desarrollo sostenible, por sentido racional, su saber debe interactuar dentro de un contexto que involucre las realidades ambientales, sociales y económicas.

Para establecer la manera como la contabilidad puede contribuir al desarrollo sostenible, se parte de que el desarrollo sostenible es de interés nacional por lo que todas las profesiones y saberes en conjunto deben propender, y de la aceptación de la definición de contabilidad que implica la ampliación del objeto de estudio 
y del propósito general de la contabilidad bajo propuesta de la T3C de Mejía, Montilla, Montes y Mora (2015) ya que, con relación al objeto de estudio la contabilidad aborda la valoración cualitativa y cuantitativa de la riqueza ambiental, social y económica controlada por las organizaciones que permite medir y brindar información para controlar aspectos de las tres realidades del desarrollo sostenible a través de la rendición de cuentas; y con relación al propósito general la contabilidad contribuye a la acumulación, generación, distribución y sostenibilidad integral de las tres riquezas controladas por las organizaciones, en otras palabras, aporta al desarrollo sostenible, generando una información útil para el interés nacional y la adecuada toma de decisiones. Esta situación genera armonía entre las tres dimensiones de un desarrollo íntegro, eliminando el reduccionismo.

Con base en lo expuesto, la contabilidad puede permitir a las organizaciones rendir cuentas respecto de las diferentes manifestaciones de riqueza controlada, a través de los modelos contables. Dichos modelos deben responder a la formulación de una estructura conceptual de la contabilidad en cada una de las dimensiones de la realidad del desarrollo sostenible. Por supuesto, los modelos contables ambientales y sociales también deben contar cada uno con criterios de reconocimiento, métodos y técnicas de medición y valoración, unidades de medida, y demás conceptos necesarios para garantizar una información útil y fiel a la realidad afectada por las organizaciones.

En síntesis, la contabilidad puede contribuir al desarrollo sostenible de la siguiente forma: a) Rompiendo los paradigmas vigentes que sólo reportan aspectos económicos y financieros. b) Definiendo un concepto de contabilidad íntegro que incluya dentro de su objeto de estudio y propósito general la contribución a un desarrollo sostenible a través de la rendición de cuentas. c) Creando modelos contables para cada una de las realidades afectadas por las organizaciones (ambiental, social, económica). d) Formulando dentro de los modelos unos criterios de reconocimiento contable para las tres realidades. e) Estableciendo métodos de medición y valoración para cada uno de los elementos que conforman los estados contables íntegros (actualmente estados financieros). f) Adoptando unidades de medida cualitativas y cuantitativas no monetarias, teniendo en cuenta las dimensiones ambientales y sociales a ser evaluadas.

A efectos de ilustrar la manera como la contabilidad puede contribuir al desarrollo sostenible se presenta la figura 1. 


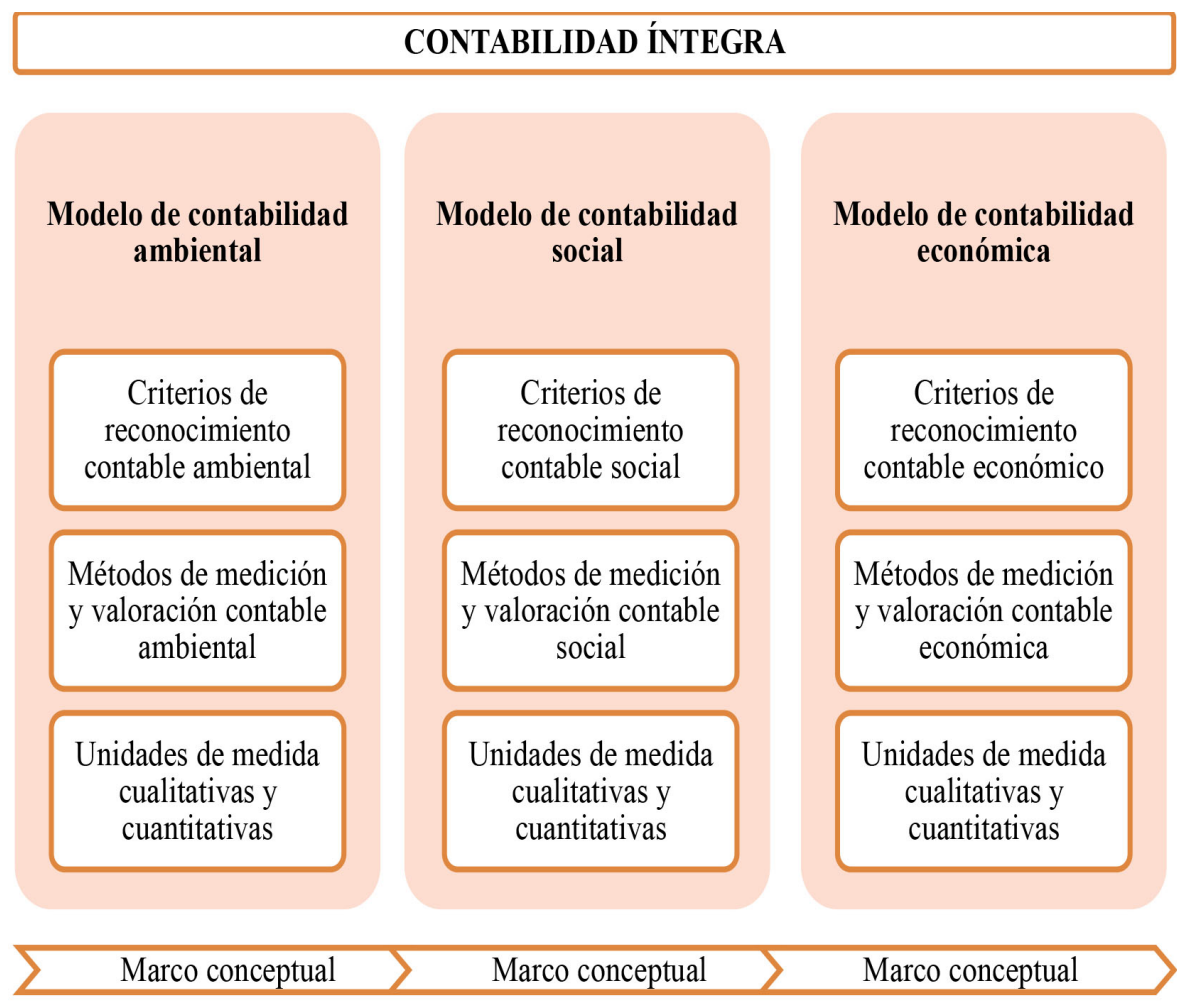

FIGURA 1

Representación de la manera en que la contabilidad puede contribuir al desarrollo sostenible Fuente: elaboración propia.

La T3C es la representación de un nuevo paradigma integrador propuesto a la comunidad científica contable, donde el desarrollo sostenible se convierte en un propósito por el que la contabilidad pretende contribuir como saber responsable que es. Así se demuestra que el contexto sobre el cual se desarrolla la contabilidad está determinado por la interacción del medio ambiente, la sociedad y la economía y que este debe primar sobre la teoría que actualmente la fundamenta. Raciocinio que permite deducir que la orientación de la contabilidad debe direccionarse hacia la solución de necesidades que involucren las tres realidades, por supuesto, desde su función de evaluar la gestión y rendir cuentas de la misma. Por lo tanto, la orientación económica determinada por la teoría clásica contable no responde a las necesidades de la totalidad del contexto sobre el que actúa la contabilidad, mientras que la propuesta emergente de la teoría tridimensional sí se fundamenta en el contexto en relación. Es decir, una orientación tridimensional que realmente ejerce como un saber estratégico al servicio de la sostenibilidad de las naciones.

\section{Discusión}

La necesidad de estudiar los paradigmas en contabilidad que impiden la incorporación de aspectos diferentes del económico resulta básicamente de tres síntomas identificados. Primero, el reduccionismo o limitación de la contabilidad como saber (solo al servicio de la economía). Segundo, la reducida investigación contable al considerar la contabilidad como un saber meramente pragmático. Tercero, la incapacidad de medición de hechos ambientales y sociales (no se controla el uso de los recursos - ineficiencia). Esto trae como consecuencia el hecho de que la contabilidad sea vista como una técnica de aplicación netamente económicofinanciera dependiente de la economía, provocando que los profesionales contables se limiten al quehacer 
de registro de información financiera, mientras en el mundo suceden crisis ambientales y sociales de difícil solución debido a que lo que no se mide no es posible controlarlo.

$\mathrm{Al}$ momento de pretender identificar por qué se presentan los síntomas mencionados y por qué se generan estas consecuencias, adquiere relevancia la fuente de origen sobre la cual se estructuran las regulaciones técnicas que rigen la contabilidad, es decir, la teoría contable aparece como el elemento referente a ser observado. Una teoría formada a través de enfoques paradigmáticos sobre los que la comunidad científica de este saber percibe la realidad de su contexto de actuación. Atendiendo a lo anterior, este estudio inicia examinando los paradigmas que están limitando o no la orientación contable solo a la dimensión económica y finaliza definiendo la verdadera orientación de la contabilidad desde su perspectiva de contribución al desarrollo sostenible.

De los resultados obtenidos es importante destacar la capacidad que tiene la contabilidad de contribuir al desarrollo sostenible y que el reconocimiento del contexto sobre el que actúa resulta ser el factor fundamental para este hecho. De aquí que el saber contable se encuentra en un debate representado desde una parte por la teoría contable clásica, de la otra parte, por la teoría tridimensional de la contabilidad. La primera ha defendido la orientación eminentemente económica, mientras que la segunda defiende una orientación de tipo tridimensional: ambiente, sociedad, economía.

La manera como la contabilidad puede contribuir al desarrollo sostenible va de la mano con la aceptación generalizada de un concepto íntegro de contabilidad por parte de la comunidad científica de este saber. Una definición que reconozca dentro del contexto sobre el que se desenvuelve, la relación del medio ambiente, sociedad y economía representa de manera categórica la integración de aspectos diferentes del económico en la contabilidad; situación que implica la formulación de una estructura teórica más amplia que incluya modelos de contabilidad ambiental y social, el establecimiento de criterios de reconocimiento para cada una de las realidades y la formulación de métodos de medición y valoración cuantitativos y cualitativos que permitan en conjunto obtener en la práctica información contable útil para la sostenibilidad integral de la riqueza controlada.

Si bien es cierta la capacidad de contribución de la contabilidad al desarrollo sostenible, también es cierta la influencia de los paradigmas contables sobre la finalidad o propósito general de este saber. De aquí que 17 de los paradigmas evaluados defienden para la contabilidad únicamente un enfoque económico, mientras que solo cuatro de ellos representan enfoques de carácter multidimensional (que relaciona al menos dos de las tres dimensiones del desarrollo sostenible). Situación que en general produce que 15 de los paradigmas no generen la incorporación de aspectos diferentes del económico, sin permitir siquiera, al menos desde su planteamiento una discusión al respecto.

Por todo lo anterior, se hace imprescindible definir la orientación de la contabilidad desde un contexto tridimensional que de manera responsable involucre todas las dimensiones de la realidad que se ven afectadas. Por supuesto, la T3C difiere con la teoría clásica de la contabilidad que ha determinado a lo largo de la historia una orientación de sentido económica. La cuestión está en determinar la totalidad del contexto sobre el que actúa el saber contable, que indiscutiblemente afecta la relación medio ambiente, sociedad, y economía y evaluar, formular o crear nuevos paradigmas que fundamenten esos nuevos aspectos.

En resumen, los síntomas identificados empiezan a ser tratados a través de la evaluación de los enfoques paradigmáticos bajo estricto sentido de carácter científico, con propuestas como la T3C, así: a) el reduccionismo o limitación del saber contable y la poca investigación se contrarrestan con el reconocimiento de la riqueza ambiental, social y económica controlada por las organizaciones como el objeto de estudio de la contabilidad. b) La incapacidad de medición de hechos ambientales y sociales se contrarresta con la incorporación de métodos de medición y valoración cuantitativas y cualitativas que permitan obtener información contable útil para tomar decisiones en pro de la sostenibilidad integral de la riqueza controlada. Dicho esto, se logra el asentamiento de unas bases teóricas que pueden referenciarse como punto de partida a futuras investigaciones. Se enriquece el contexto de actuación del saber contable, su capacidad de contribución 
al desarrollo sostenible, la influencia de los paradigmas adoptados, su orientación tridimensional, entre otros más aspectos que resultan importantes siempre que se pretenda investigar en relación al objeto de estudio de la contabilidad, su función y su propósito general.

\section{Conclusiones}

La contabilidad es instrumentalizada desde una corriente eminentemente económica, ya que los paradigmas existentes para este saber son esencialmente fundamentados por la teoría contable clásica que es reduccionista e impide la incorporación de la dimensión ambiental y social según las características estudiadas en aspectos del enfoque, base del sustento, actualización y efectos que genera su aplicación a diversas realidades del contexto.

La mayoría de los paradigmas evaluados no permiten la incorporación de aspectos diferentes al económico de acuerdo con los cuatro criterios estudiados. El enfoque es principalmente económico o reduccionista; la base del sustento proclama totalmente realidades contundentes, pero en relación al enfoque económico actual al que responde (Contabilidad clásica); el nivel de actualización revela que siguen vigentes los paradigmas estudiados desde hace 46 años (1974-2020) sin actualizarse a las necesidades del contexto; y los efectos de la aceptación de los paradigmas existentes impide o limita la posibilidad de incorporar aspectos de la dimensión ambiental y social.

Los paradigmas estudiados que sí permiten la incorporación de aspectos diferentes al económico son de enfoque multidimensional; de ellos la mayoría reconocen la realidad social y económica. La base del sustento proclama realidades con relación al enfoque económico, pues el sistema contable que se aplica al aspecto social es el de Contabilidad Social que mantiene el enfoque reduccionista y no el de Sociocontabilidad. En el nivel de actualización todos los paradigmas se encuentran vigentes, siendo el paradigma emergente de la T3C el más reciente y el único que agrupa las tres dimensiones que interactúan para promover el desarrollo sostenible.

Categóricamente la contabilidad puede contribuir al desarrollo sostenible una vez reflexione sobre sus enfoques paradigmáticos que la enmarcan solamente a la orientación económica. Su manera de contribuir está representada en la estructuración de una contabilidad íntegra. Situación que supone la creación de un modelo contable ambiental, un modelo contable social y un modelo contable económico; cada uno con sus propios criterios de reconocimiento y métodos de medición y valoración con unidades de medida cualitativas y cuantitativas presentadas en un marco conceptual elaborado, presentado y adoptado por parte de la comunidad científica contable.

La orientación de la contabilidad es discutida desde dos tendencias: la orientación económica respaldada por la teoría contable clásica y la orientación tridimensional respaldada por la teoría tridimensional de la contabilidad T3C. Sin embargo, más allá de que la orientación económica de la concepción clásica de teoría contable sea la mayormente aceptada y adoptada por la comunidad científica de este saber en la actualidad, es irresponsable reconocer que su contexto de actuación no es evidentemente influenciado por la relación sociedad, medio ambiente y economía. Es por esto que la orientación de la contabilidad es de origen tridimensional más allá de la teoría que la propone. No se trata de que la contabilidad sea el saber que se encargue de estudiar los aspectos ambientales y sociales, sino que como saber responsable que es, contribuya de la manera más eficiente a los propósitos de una sostenibilidad integral.

La óptica del saber contable desde una multiplicidad de paradigmas de Belkaoui o la óptica de matriz disciplinar de Kuhn adquieren sentido para la contabilidad, teniendo en cuenta que su comunidad científica genera conocimiento en torno a diferentes corrientes que buscan la aceptación general de determinada orientación para el saber contable. El paradigma de la ganancia líquida (o beneficio verdadero) y el paradigma de utilidad para la toma de decisiones son los grandes agrupadores de los diferentes enfoques paradigmáticos estudiados, es decir representan la gran matriz disciplinar. 
Los paradigmas existentes tienen en su mayoría un enfoque económico situación que limita la capacidad de respuesta de la contabilidad como saber a las tres realidades que le conciernen. Esta situación conlleva a evaluar la necesidad de crear nuevos paradigmas para el aspecto social y ambiental que permitan analizar las realidades a través de la propuesta de matriz disciplinar articulando diferentes paradigmas para la solución de problemas concretos. De esta manera, la sola evaluación del enfoque que representan abre un debate que puede resultar en la ampliación de un contexto producto de líneas de investigación encaminadas a la construcción teórica de conceptualización, medición, valoración, registro, revelación y control para la dimensión ambiental y social.

Como limitación de este estudio se encuentra que la comunidad científica contable no maneja un concepto unificado para los enfoques paradigmáticos lo que dificulta su alineación bajo un mismo criterio y supone superposiciones encontradas, tampoco cuenta con un enfoque paradigmático claramente definido siempre que vive del constante debate entre lo individual, lo múltiple y lo integrador.

\section{Consideraciones éticas}

La investigación no requirió un aval ético al ser una revisión documental que no genera afectación y se sustenta en las fuentes referenciadas.

\section{Contribución de los autores}

Los dos autores contribuyeron en la investigación con la recolección, revisión, análisis y discusión de la información teórica empleada en este estudio.

\section{Conflictos de interés}

Los autores no tienen ningún tipo de conflicto de interés asociado al desarrollo de la investigación.

\section{Agradecimientos}

La investigación no contó con ningún tipo de financiación para su elaboración.

\section{Referencias}

Amigo, A. (2018). ¿Qué es la contabilidad social y ambiental? Revista Activos, 16(30), 127-152. https://revistas.usan totomas.edu.co/index.php/activos/article/download/5063/pdf

Ariza, D. (2000). Una perspectiva para captar la inserción contable en la problemática medio ambiental. Revista LEGIS del Contador, 4, 161-191. https://xperta.legis.co/visor/temp_rcontador_253a7623-db78-4d00-8bec-6040694 41254

Belkaoui, A. R. (1993). Accounting theory. Londres: Hartcourt Brace.

Bunge, M. (1985). La investigación cientifica: su estrategia y su filosofía. Bogotá: Ariel.

Bustillo, L., \& Martínez, J. (2008). Los enfoques del desarrollo sustentable. Interciencia, 33(5), 389-395. https://ww w.redalyc.org/pdf/339/33933512.pdf

Casal, R., \& Viloria, N. (2007). La ciencia contable, su historia, filosofia, evolución y su producto. Actualidad Contable FACES, 10(15), 19-28. https://www.redalyc.org/articulo.oa?id=25701503

Daros, W. R. (2007). Los condicionamientos sociales en los paradigmas científicos: Popper y Kuhn. Invenios: Revista de investigación académica, 18, 47-74. https://dialnet.unirioja.es/servlet/articulo?codigo $=4263832$ 
García, L. (2008). Aproximación epistemológica al concepto de ciencia: una propuesta básica a partir de Kuhn, Popper, Lakatos y Feyerabend. Revista Andamios, 4(8), 185-212. doi:http://dx.doi.org/10.29092/uacm.v4i8.307

García, C. L. (1997). Naturaleza de la contabilidad. Contabilidad y Auditoría, 3(5), 13-37. http://www.ojs.econ.uba .ar/index.php/Contyaudit/article/view/212

García, C. L. (2002). El problema del uso de modelos en la contabilidad. Revista Internacional LEGIS de Contabilidad y Auditoría, 12, 199-235. http://legal.legis.com.co/document/Index?obra=rcontador\&document=rcontador_ 7680752a7d70404ce0430a010151404c

Hendriksen, E. S. (1974). Teoria de la contabilidad. México DF: UTEHA.

Kuhn, T. S. (1962). The Structure of Scientific Revolutions. Chicago: University Of Chicago Press.

Mantilla, S., \& Tristancho, G. (1997). Conocimiento, metodología e investigación contable. Cali: Roesga.

Marín, L. F. (2007). La noción de paradigma. Signo y Pensamiento, 26(50), 34-45. https://revistas.javeriana.edu.co/i ndex.php/signoypensamiento/article/view/3705

Marín, J. D. (2007). Del concepto de paradigma en Thomas S. Kuhn, a los paradigmas de las Ciencias de la cultura. Revista Magistro, 1(1), 73-88. https://dialnet.unirioja.es/servlet/articulo?codigo=4038923

Martínez, G. L. (2007). Los paradigmas contables: La borrosa impronta de una interpretación epistemológica. Lúmina, $8,205-234$.

Mejía, E. (2014). Biocontabilidad: Hacia una definición. Lúmina, 15, 106-129. https://www.google.com/url?hl=es\&q =http://revistasum.umanizales.edu.co/ojs/index.php/Lumina/article/download/1069/1174\&sa=D\&ust=15 88632064131000\&usg=AFQjCNHDYJPW3DfJEeadAsEjtXMmmeduqA

Mejía, E. (2016). Contabilidad Tridimensional. Contabilidad para la Sostenibilidad. Armenia, Quindío, Colombia: Ponencia. Universidad la Gran Colombia seccional Armenia.

Mejía, E., \& Montes, C. A. (2009). Enfoque crítico de la orientación socioambiental en la profesión contable. Revista internacional LEGIS de Contabilidad y Auditoria, 39, 149-177. http://legal.legis.com.co/document/Index?obr $\mathrm{a}=$ rcontador\&document $=$ rcontador_7680752a7dfa404ce0430a010151404c

Mejía, E., Montes, C. A., \& Mora, G. (2013). Estructura conceptual de la teoría tridimensional de la contabilidad. Contexto, 2(1), 49-70. https://revistas.ugca.edu.co/index.php/contexto/article/view/42/338

Mejía, E., Montes, C. A., \& Mora, G. (2013). Identific0ción del objeto de estudio de la Contabilidad. Libre Empresa, 10(1), 51-70. https://revistas.unilibre.edu.co/index.php/libreempresa/article/view/2985/2396

Mejía, E., Montilla, O., Montes, C. A., \& Mora, G. (2014). Teoría tridimensional de la Contabilidad T3C (Versión 2.0): Desarrollos, avances y temas propuestos. Libre Empresa, 22, 95-120. https://doi.org/10.18041/1657-281 5/libreempresa.2014v1 ln2.3027

Mejía, E., Montilla, O. d., Montes, C. A., \& Mora, G. (2015). Teoría Tridimensional de la Contabilidad T3C - Versión 2.0. Pereira: Universidad Libre Seccional Pereira.

Montes, C. A., Mejía, E., \& Valencia, J. J. (2006). Paradigmas en contabilidad, Cali, Colombia: Artes Gráficas del Valle.

Organización de las Naciones Unidas - ONU (1983). Nuestro futuro común. Comisión Mundial sobre el Medio Ambiente y el Desarrollo.

Santos, G., Durán G, M., Urrea Bello, L. M., \& Urquijo, W. F. (1997). Acerca de los “paradigmas contables”. Innovar, 9, 141-150. https://revistas.unal.edu.co/index.php/innovar/article/view/19311/20262

Tua P., J. (2012). Contabilidad y desarrollo económico. Contabilidad y Negocios, 7(13), 94-110. https://www.redaly c.org/articulo.oa?id=281623577008

Tua P., J. (1996). Globalización y regulación contable: algunos retos para nuestra profesión en América Latina. ICAC.

Uribe, M. V. (2014). Caracterización de la evolución en el concepto de contabilidad de 1990 a 2010. Cuadernos de Contabilidad, 15(37), 239-259. http://www.scielo.org.co/pdf/cuco/v15n37/v15n37a10.pdf

Viveros, E. F. (2015). Sobre la transición de la noción de paradigma a la de matriz disciplinaria en Thomas. Revista Virtual Universidad Católica del Norte, (44), 163-174. https://www.redalyc.org/articulo.oa?id=194238608012

Zarta, P. (2018). La sustentabilidad o sostenibilidad: un concepto poderoso para la humanidad. Revista de Humanidades Tabula Rasa, 28, 409-423.https://www.redalyc.org/ar 


\section{Notas}

* Artículo de investigación.

Licencia Creative Commons CC BY 4.0

Para citar este artículo: Rivera Q., V., \& Henao P., J. J. (2021). Paradigmas en contabilidad que impiden la incorporación de aspectos diferentes del económico. Cuadernos de Contabilidad, 22. https://doi.org/10.11 144/Javeriana.cc22.pcii 